Classification

Physics Abstracts

$43.00-72.00$

\title{
Génération d'ondes acoustiques dans le silicium par faisceau d'électrons ou laser modulé
}

\author{
D. Marty-Dessus ( $\left.{ }^{1}\right)$, M. Ez Zejjari $\left({ }^{1}\right)$, N. Boughanmi $\left({ }^{1,2}\right)$, J. Boucher $\left({ }^{2}\right)$ et \\ J. L. Franceschi $(1,2)$ \\ (1) CEMES-LOE, CNRS, 29 rue Jeanne Marvig, 31055 Toulouse Cedex, France \\ $\left({ }^{2}\right)$ Institut National Polytechnique, ENSEEIHT, 2 rue Camichel, 31071 Toulouse Cedex, France \\ (Reçu le 7 novembre 1991, révisé le 9 mars 1992, accepté le 30 mars 1992)
}

\begin{abstract}
Résumé. - Une méthode d'imagerie sur objets massifs est proposée. L'impact d'un faisceau modulé d'électrons ou laser génère des ondes acoustiques dans un solide. Dans le silicium, la création d'un gradient thermique et d'une population de porteurs en excès est à l'origine de l'apparition d'ondes élastiques. Nous proposons une modélisation de ces deux phénomènes pour dégager leur importance relative sur le signal acoustique total et analyser l'influence des caractéristiques générales du semiconducteur (durée de vie des porteurs, vitesse de diffusion électronique...). Nous mettons en évidence l'intérêt d'une telle méthode pour la détection des défauts en subsurface dans le silicium.
\end{abstract}

Abstract. - An imaging system placed on a bulk specimen is proposed. A modulated electron or laser beam acts as an acoustic waves source in a bulk specimen. In silicon, the creation of a thermal gradient and an excess carrier population is at the origin of elastic waves generation. We propose here a modelisation of these two phenomena in order to emphasize their relative importance on the total acoustic signal and the influence of the semiconductor general characteristics as carrier lifetimes or electronic diffusivity. We point out the utility of this method for the detection of subsurface defects in silicon.

\section{Introduction.}

L'imagerie utilisant les phénomènes acoustiques est de plus en plus utilisée en contrôle non destructif. En effet, ces techniques s'adaptent de mieux en mieux aux impératifs industriels qui visent à contrôler la qualité d'un objet, souvent volumineux, sans prélèvement d'échantillons $[1,2]$.

Dans le cadre de notre étude, nous nous proposons de contrôler des échantillons particuliers, des circuits silicium. Il ne s'agit donc plus de contrôle non destructif classique car

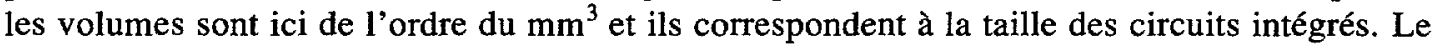
but est de réaliser l'analyse et l'imagerie sélective en profondeur d'éventuels défauts dans la structure silicium par une méthode non destructive. 


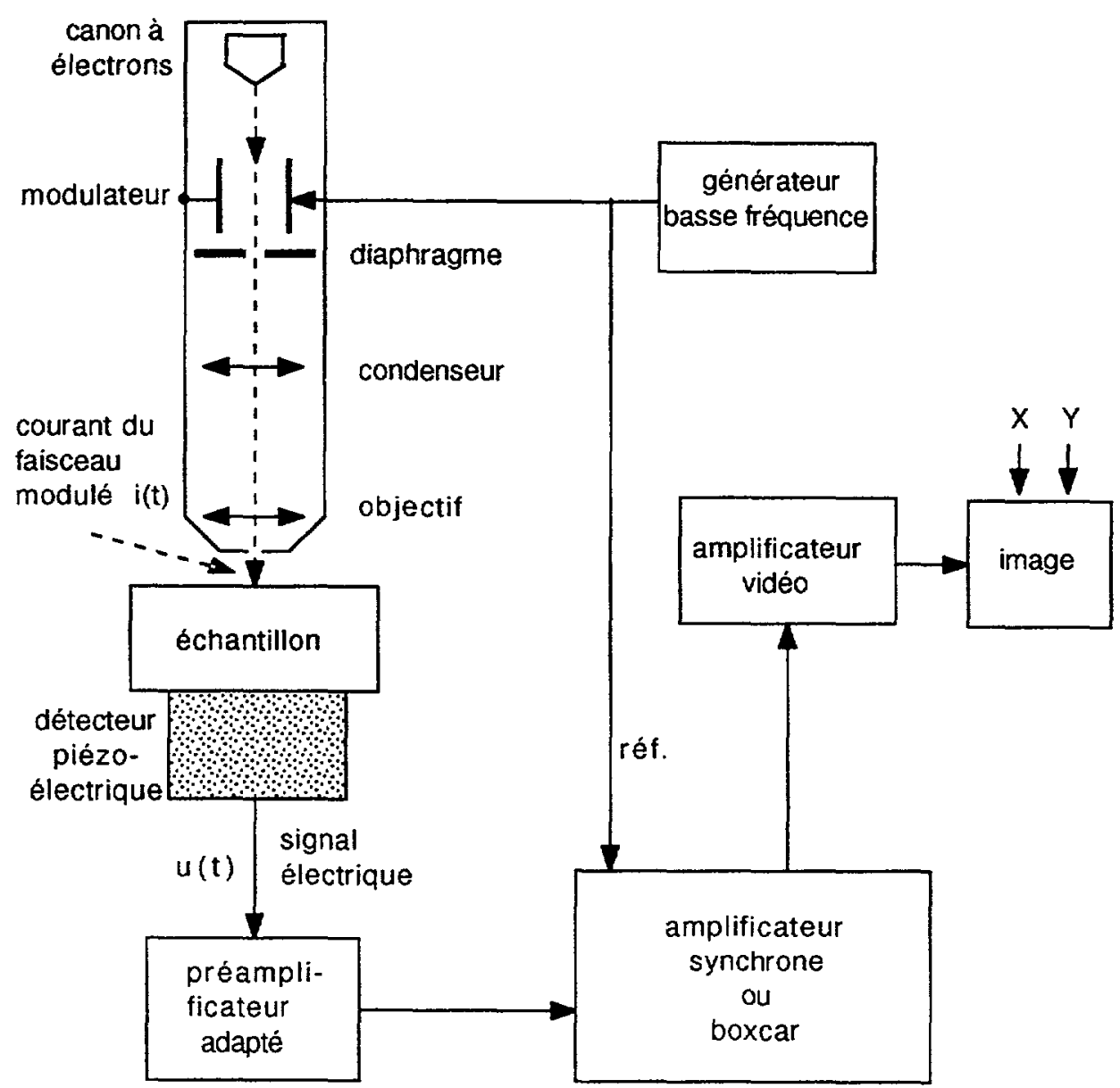

Fig. 1. - Schéma fonctionnel du système d'imagerie acousto-électronique.

[Block diagram of the acousto-electronic imaging system.]

La technique que nous utilisons est basée sur la mise en œuvre d'un mini microscope électronique transportable (MEBIS [3] : Microscope Electronique à Balayage « in situ »). La colonne électro-optique, petite et légère, peut être placée juste au-dessus de l'échantillon. Avec un système de contrôle spécialement adapté, le faisceau d'électrons modulé en intensité est utilisé pour générer des ondes acoustiques. En combinant le signal acoustique avec ce faisceau, des images de la subsurface du matériau sont réalisables.

En pratique, suivant le mode de détection utilisé (amplificateur synchrone ou Boxcar) et le type de manipulation souhaitée (analyse temporelle du signal transitoire par exemple), le faisceau est modulé soit en signaux carrés soit en impulsions.

Pour prévoir l'évolution du signal acoustique en fonction de différents paramètres et après l'étude de différents métaux [4], il a fallu prendre en compte divers phénomènes physiques qui apparaissent dans le silicium soumis à un faisceau d'électrons ou de lumière modulée :

- génération d'ondes dues à l'apparition d'un gradient thermique ;

- génération due à une création de porteurs en excès. 
L'étude théorique que nous proposons permet de simuler ces deux phénomènes pour essayer de dégager leur importance relative sur le signal résultant en fonction de la fréquence de modulation du faisceau, ainsi que l'influence de la durée de vie des porteurs sur l'amplitude des ultrasons. Il sera alors possible de prévoir suivant les conditions d'expérimentation sur quels paramètres il faut agir pour obtenir un signal maximum et réaliser l'imagerie dans de bonnes conditions.

Dans une deuxième partie nous exposerons les avantages d'une telle méthode pour la détection des défauts dans les semiconducteurs.

\section{Les phénomènes physiques de génération d'ondes ultrasonores.}

2.1 Couplage thermigue, COUPlage PaR eXCÈs DE PORTEurs. - La génération du son par faisceau d'électrons (ou LASER) a été expliquée par plusieurs auteurs $[5,6]$ comme la conversion d'ondes thermiques générées par le chauffage de l'échantillon en ondes acoustiques. L'apparition d'un gradient de température provoque en effet une dilatation thermique du matériau, l'échantillon se déforme et il y a donc création d'ultrasons.

Aux fréquences de modulation utilisées $(<1 \mathrm{Mhz}$ ), la longueur d'onde acoustique (de quelques $\mathrm{mm}$ à quelques $\mathrm{cm}$ ) n'intervient pas dans la résolution. Ces ondes jouent uniquement le rôle de porteuses d'informations générées par le chauffage de l'échantillon.

Il a été prouvé que le phénomène thermique est prépondérant dans la majorité des matériaux, par exemple les métaux [7]. Cependant certaines expériences récentes ont montré qu'il pouvait devenir négligeable devant d'autres phénomènes, notamment dans les semiconducteurs [8].

En effet, un faisceau d'énergie suffisante crée une population de porteurs (électrons et trous) en excès dans l'échantillon. Cette densité de porteurs va d'une part contribuer au processus de diffusion thermique, mais aussi provoquer par sa présence une déformation du tenseur de permittivité du matériau. Le cristal va se déformer ce qui provoque une deuxième source d'émission acoustique. Ces deux phénomènes (couplage thermique et couplage par excès de porteurs) ne sont pas indépendants [9]. Dans un premier temps, il va exister une thermalisation de certains porteurs au-delà de la bande interdite du matériau, donc un effet sur la diffusion de la température. Ce phénomène, supposé quasiment instantané, est à séparer d'un autre beaucoup plus lent, qui consiste en la recombinaison non radiative d'autres porteurs qui ont diffusé dans le substrat. Il est donc nécessaire d'inclure l'effet des porteurs en excès dans l'équation de diffusion de la température.

Ceci a été clairement mis en évidence par Stearns [10] et Fournier [11] qui ont prouvé dans le cas du silicium que la prise en compte du seul phénomène thermique pour la modélisation de la génération du son n'est pas valable.

2.2 EXPRESSION DES DISTRIBUTIONS DE TEMPÉRATURE ET DE PORTEURS POUR UNE EXCITATION LASER. - Pour pouvoir évaluer la contribution de chaque phénomène au signal total, il est nécessaire d'établir les expressions des distributions de température et des porteurs créés. Les hypothèses relatives aux caractéristiques de la source et du point éclairé sont les suivantes :

- intensité $I_{0}$. densité de puissance du faisceau supposée constante sur le spot focalisé

- $P_{0}=(1-R) I_{0}$. densité de puissance absorbée à la surface de l'échantillon avec $R$ coefficient de réflexion optique du silicium

- la source est de faible intensité c'est-à-dire que nous n'atteignons pas le régime d'ablation et que nous pouvons supposer la linéarité de l'équation (3) de diffusion des porteurs minoritaires 


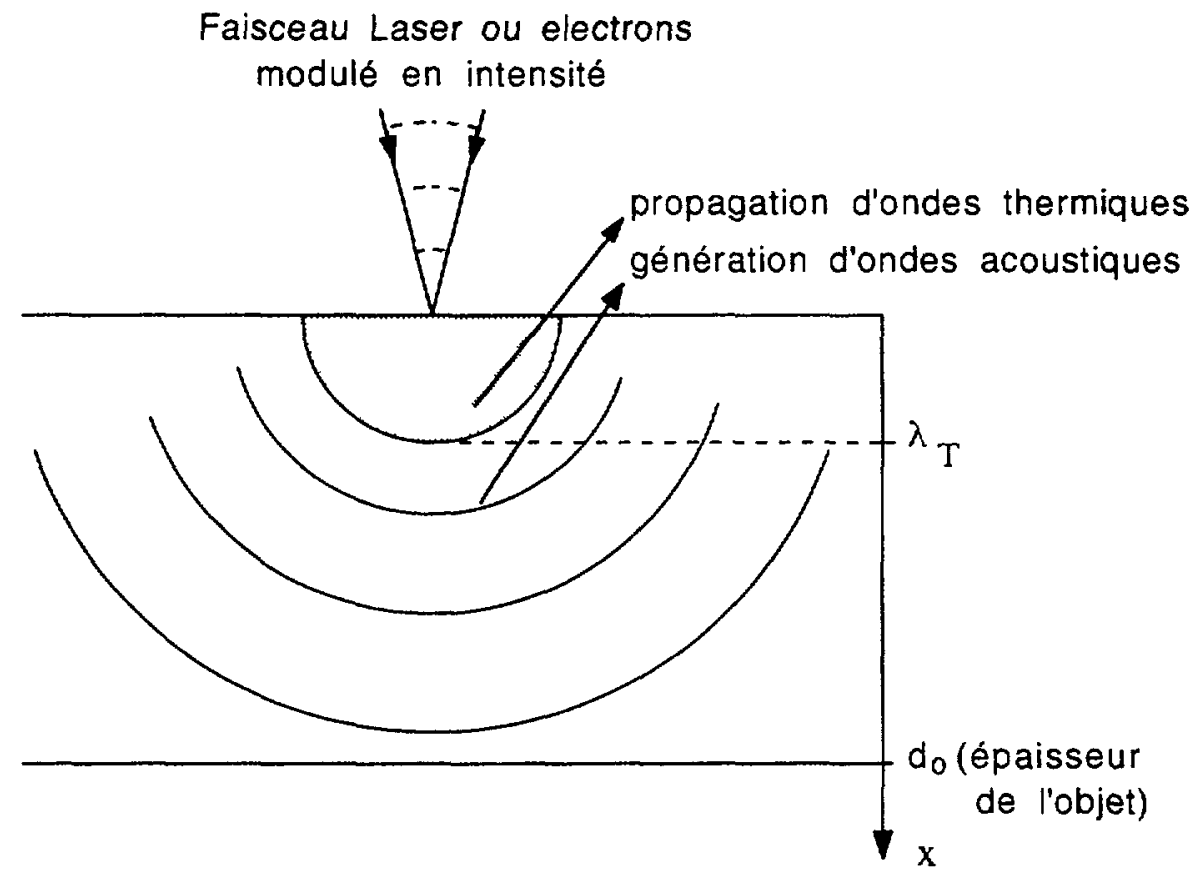

Fig. 2. - Génération des ondes acoustiques.

[Generation of acoustic waves.]

- le rendement quantique est supposé égal à 1

- l'échantillon est « opaque », l'absorption optique se fait en surface.

Une paire électron-trou créée par le faisceau d'énergie suffisante transfère immédiatement son énergie à la structure. Celle-ci est égale à $\Delta E=E_{0}-E_{\mathrm{G}}$ où $E_{0}$ est l'énergie de la particule incidente et $E_{\mathrm{G}}$ le gap du matériau. La puissance émise est donc :

$$
\left(\frac{\Delta E P_{0}}{E_{0}}\right) \text {. }
$$

Dans un deuxième temps et beaucoup plus lentement, un transfert d'énergie s'opère par recombinaison non radiative de certains porteurs qui ont diffusé dans le substrat, ce qui correspond à une puissance $\left(E_{\mathrm{G}} \frac{N}{\tau}\right), N$ étant la densité de porteurs créés et $\tau$ la durée de vie des porteurs minoritaires.

Nous pouvons donc modéliser la distribution thermique en supposant une vitesse de recombinaison en surface nulle ainsi qu'en négligeant l'effet des porteurs majoritaires, par l'équation:

$$
D_{\mathrm{T}} \frac{\partial^{2} T(x, t)}{\partial x^{2}}=\frac{\partial T(x, t)}{\partial t}-\frac{E_{\mathrm{G}} D_{\mathrm{T}}}{k} \frac{N(x, t)}{\tau}
$$

avec la condition à la limite :

$$
-\left.K \frac{\partial T}{\partial x}\right|_{x=0}=\frac{\Delta E P_{0}}{E_{0}} \mathrm{e}^{\imath \omega t}
$$


où :

$D_{\mathrm{T}} \quad$ vitesse de diffusion thermique $\left(\mathrm{cm}^{2} / \mathrm{s}\right)$

$K$ conductivite thermique $\left(\mathrm{W} / \mathrm{cm} .{ }^{\circ} \mathrm{C}\right)$

$E_{0} \quad$ énergie de la particule incidente $(\mathrm{J})$

$P_{0} \quad$ densité de puissance absorbée $\left(\mathrm{W} / \mathrm{cm}^{2}\right)$

$\omega / 2 \pi$ fréquence de modulation faisceau $(\mathrm{Hz})$

$\tau$ durée de vie des minoritaires (s).

Pour modéliser le comportement des porteurs minoritaires, considérons l'équation de diffusion :

$$
D_{\mathrm{E}} \frac{\partial^{2} N(x, t)}{\partial x^{2}}=\frac{\partial N(x, t)}{\partial t}+\frac{N(x, t)}{\tau}
$$

avec la condition à la limite

$$
D_{\mathrm{E}} \frac{\partial N(x, t)}{\partial x}=-\frac{P_{0} \mathrm{e}^{t \omega t}}{E_{0}}
$$

avec $D_{\mathrm{E}}=$ vitesse de diffusion électronique $\left(\mathrm{cm}^{2} / \mathrm{s}\right)$.

Fournier et al. [11] ont proposé une résolution de ces équations en tenant compte du fait que les équations (1) et (3) sont couplées par le terme de recombinaison des porteurs. Ils ont donné une solution générale de $T(x, t)$ et $N(x, t)$ de la forme :

$$
\begin{gathered}
T(x, t)=\left\{\frac{P_{0} E_{\mathrm{G}}\left(\lambda_{\mathrm{T}} \mathrm{e}^{-x / \lambda}-\lambda_{\mathrm{E}} \mathrm{e}^{-x / \lambda_{\mathrm{E}}}\right)}{E_{0} \tau D_{\mathrm{E}} K\left(1 / \tau D_{\mathrm{E}}+i \omega\left(1 / D_{\mathrm{E}}-1 / D_{\mathrm{T}}\right)\right.}+\left(\frac{E_{0}-E_{\mathrm{G}}}{E_{0} K}\right) P_{0} \lambda_{\mathrm{T}} \mathrm{e}^{-x / \lambda_{\mathrm{T}}}\right\} \mathrm{e}^{i \omega t} \\
N(x, t)=\left(\frac{\lambda_{\mathrm{E}} P_{0}}{E_{0} D_{\mathrm{E}}} \mathrm{e}^{-x / \lambda_{\mathrm{E}}}\right) \mathrm{e}^{i \omega t}
\end{gathered}
$$

où

$$
\lambda_{\mathrm{T}}=\sqrt{\frac{\overline{D_{\mathrm{T}}}}{i \omega}} \quad \text { et } \quad \lambda_{\mathrm{E}}=\sqrt{\frac{D_{\mathrm{E}} \tau}{1+i \omega \tau}}
$$

avec

$\lambda_{\mathrm{T}} \quad$ longueur de diffusion thermique complexe

$\lambda_{\mathrm{E}} \quad$ longueur de diffusion-recombinaison complexe [12].

Ces expressions de $T$ et $N$ vont nous permettre de modéliser la génération et la propagation des ultrasons.

\section{Génération et propagation des ondes acoustiques.}

3.1 InTRODUCTION. - Dans un solide, toute contrainte $\sigma_{\imath \jmath}$ provoque une déformation $e_{i j}$ telle que :

$$
e_{i j}=S_{i j k \ell} \sigma_{k \ell}
$$

où

$\sigma_{i j}=$ tenseur de contrainte

$e_{i j} \quad=$ tenseur de déformation

$S_{\imath j k \ell}=$ tenseur de souplesse élastique. 
En négligeant les forces extérieures sur le solide, l'équation du mouvement acoustique s'écrit [13] :

$$
\nabla . \sigma=\rho \frac{\partial^{2} u}{\partial t^{2}}
$$

où $u$ est le déplacement mécanique et $\rho$ la masse volumique du matériau. Nous allons appliquer cette équation au cas d'une contrainte thermique seule, puis d'une contrainte électronique. Enfin, nous donnerons une évaluation du déplacement total résultant de la simultanéité des deux phénomènes.

Nous nous intéressons à un seul type d'ondes acoustiques, les ondes de volume longitudinales et transversales. Pour simplifier l'étude théorique, seules les ondes longitudinales suivant l'axe $x$ sont prises en compte dans la résolution.

Les modes propres de vibration de l'objet peuvent moduler le signal acoustique en introduisant des variations spatiales parasites dans son amplitude et dans sa phase. Pour ne pas avoir une diminution de ce signal par interférences destructrices et pour ne pas masquer les effets de contraste provenant des variations locales des paramètres thermoélastiques, il faut travailler à des fréquences de modulation telles que la surface du capteur couvre une seule région correspondant à un maximum (ventre) de vibration. Ceci s'obtient simplement à des fréquences faibles. Pour des fréquences plus élevées, l'utilisation de capteurs à surface de contact plus réduite s'impose. C'est ainsi que l'on peut s'affranchir des modes propres de vibration de l'objet.

3.2 Couplage THERMique. - Une distribution de température locale non uniforme dans un solide produit une déformation additionnelle du type :

$$
e_{\mathrm{T}}=\alpha T(x, t)
$$

où $\alpha$ : coefficient linéaire de dilatation thermique.

Cette deformation est telle que

$$
\tau=C\left(e_{\mathrm{M}}-e_{\mathrm{T}}\right)
$$

où $C=$ tenseur de rigidité.

La contrainte résultante est donc :

$$
\sigma=\rho v^{2} e_{\mathrm{M}}-E_{\mathrm{el}} \alpha T(x, t)
$$

où $E_{\mathrm{el}}=$ module élastique

et $v=$ vitesse longitudinale du son

en reportant cette équation dans celle du mouvement (7) et en tenant compte du fait que

$$
e_{\mathrm{M}}(x, t)=\frac{\partial u(x, t)}{\partial x}
$$

nous obtenons

$$
\frac{\partial^{2} u}{\partial t^{2}}=v^{2} \frac{\partial^{2} u}{\partial x^{2}}-\frac{\alpha E_{\mathrm{el}}}{\rho} \frac{\partial T(x, t)}{\partial x}
$$

En se limitant au terme fondamental de pulsation $\omega$ du signal dû à une modulation en 
$\mathrm{e}^{i \omega t}$, nous pouvons séparer les variables $x$ et $t$, ce qui donne :

$$
v^{2} \frac{\partial^{2} u(x, t)}{\partial x^{2}}+\omega^{2} u(x, t)=\frac{\alpha E_{\mathrm{el}}}{\rho} \cdot \frac{\partial T(x, t)}{\partial x}
$$

En reportant la valeur de $T(x, t)$ de l'équation (5), nous obtenons :

$$
v^{2} \frac{\partial^{2} u(x, t)}{\partial x^{2}}+\omega^{2} u(x, t)=\beta_{1} \mathrm{e}^{-x / \lambda_{\mathrm{E}}}+\beta_{2} \mathrm{e}^{-x / \lambda_{\mathrm{T}}}
$$

La solution générale de cette équation est de la forme :

$$
u(x, t)=\left(C_{1} \mathrm{e}^{-i k x}+C_{2} \mathrm{e}^{+i k x}+C_{3} \mathrm{e}^{-x / \lambda_{\mathrm{E}}}+C_{4} \mathrm{e}^{-x / \lambda} \mathrm{T}\right) \mathrm{e}^{i \omega t}
$$

avec:

$$
\begin{gathered}
C_{3}=\frac{\beta_{1}}{\omega^{2}+\frac{v^{2}}{\lambda_{\mathrm{E}}^{2}}} \quad C_{4}=\frac{\beta_{2}}{\omega^{2}+\frac{v^{2}}{\lambda_{\mathrm{T}}^{2}}} \\
\beta_{1}=\frac{\alpha E_{\mathrm{el}}}{\rho}\left(\frac{a}{b+i c}\right) \quad \beta_{2}=\left(-h-\frac{a}{b+i c}\right) \frac{\alpha E_{\mathrm{el}}}{\rho} \\
a=\frac{P_{0} E_{\mathrm{G}}}{E_{0} D_{\mathrm{E}} \tau K} \quad b=\frac{1}{\tau D_{\mathrm{E}}} \quad c=\omega\left(1 / D_{\mathrm{E}}-1 / D_{\mathrm{T}}\right) \quad h=\frac{\left(E_{0}-E_{\mathrm{G}}\right) P_{0}}{E_{0} K} \\
k=\frac{\omega}{v}
\end{gathered}
$$

En supposant la surface de l'échantillon libre de contrainte (en $x=0$ ) et en faisant l'hypothèse d'un régime de plaque, c'est-à-dire l'objet d'épaisseur $d_{0}$ est libre de contrainte en $x=d_{0}$, nous introduisons les conditions aux limites :

$$
\sigma(x=0)=\sigma\left(x=d_{0}\right)=0
$$

qui nous permet de calculer $C_{1}$ et $C_{2}$ (cf. Annexe).

Nous pouvons ainsi évaluer le déplacement sur la face arrière de l'échantillon en $x=d_{0}$.

$$
\begin{aligned}
& u\left(d_{0}\right)= \\
& =\frac{\left\{\left(A^{\prime}+\frac{C_{3}}{\lambda_{\mathrm{E}}}\right)+\left(A^{\prime \prime}+\frac{C_{4}}{\lambda_{\mathrm{T}}}\right)\right\}-\left\{\left(A^{\prime}+\frac{C_{3}}{\lambda_{\mathrm{E}}}\right) \mathrm{e}^{-d_{0} / \lambda_{\mathrm{E}}}+\left(A^{\prime \prime}+\frac{C_{4}}{\lambda_{\mathrm{T}}}\right) \mathrm{e}^{-d_{0} / \lambda_{\mathrm{T}}}\right\} \cos k d_{0}}{k \sin k d_{0}}+ \\
& +C_{3} \mathrm{e}^{-d_{0} / \lambda_{\mathrm{E}}}+C_{4} \mathrm{e}^{-d_{0} / \lambda_{\mathrm{T}}}
\end{aligned}
$$

pour un échantillon tel que $d_{0} \gg \lambda_{\mathrm{E}}$ et $d_{0} \gg \lambda_{\mathrm{T}}$, ce qui est toujours le cas pour des objets dont l'épaisseur est de l'ordre du mm, en posant $\cos k d_{0}=\cos \frac{\omega}{v} d_{0} \approx 1$, soit encore $\sin k d_{0} \approx k d_{0}$, $u\left(d_{0}\right)$ se réduit à :

$$
u\left(d_{0}\right)=\frac{\left(A^{\prime}+\frac{C_{3}}{\lambda_{\mathrm{E}}}\right)+\left(A^{\prime \prime}+\frac{C_{4}}{\lambda_{\mathrm{T}}}\right)}{k^{2} d_{0}}+C_{3} \mathrm{e}^{-d_{0} / \lambda_{\mathrm{E}}}+C_{4} \mathrm{e}^{-d_{0} / \lambda_{\mathrm{T}}}
$$


En remplaçant $C_{3}$ et $C_{4}$ par (13), on obtient :

$$
u\left(d_{0}\right)=A^{\prime}\left(\frac{\lambda_{\mathrm{E}}^{2}}{d_{0}}-\lambda_{\mathrm{E}} \mathrm{e}^{-d_{0} / \lambda_{\mathrm{E}}}\right)+A^{\prime \prime}\left(\frac{\lambda_{\mathrm{T}}^{2}}{d_{0}}-\lambda_{\mathrm{T}} \mathrm{e}^{-d_{0} / \lambda_{\mathrm{T}}}\right)
$$

soit encore en évaluant les différents termes :

$$
u\left(d_{0}\right)_{\mathrm{T}}=\frac{A^{\prime} \lambda_{\mathrm{E}}^{2}+A^{\prime \prime} \lambda_{\mathrm{T}}^{2}}{d_{0}}
$$

en remplaçant $A^{\prime}$ et $A^{\prime \prime}$ par leurs valeurs respectives, nous obtenons :

$$
\left|u\left(d_{0}\right)\right|_{\mathrm{T}}=\frac{\alpha E_{\mathrm{el}}}{\rho v^{2} d_{0}}\left|\frac{-a}{b+i c}\left\{\left(\frac{D_{\mathrm{E}} \tau}{1+i \omega \tau}\right)^{3 / 2}-\left(\frac{D_{\mathrm{T}}}{i \omega}\right)^{3 / 2}\right\}+h\left(\frac{D_{\mathrm{T}}}{i \omega}\right)^{3 / 2}\right| .
$$

3.3 COUPLAGE PAR EXCÈS DE PORTEURS. - Le faisceau primaire crée une population de porteurs dans le matériau provoquant une déformation du tenseur de permittivité de l'échantillón qui va se « déformer ». Dans le cas d'une excitation laser, cette déformation est de la forme $[10]$ :

$$
e_{\mathrm{E}}=\frac{1}{3}\left(\frac{\mathrm{d} E_{\mathrm{G}}}{\mathrm{d} P}\right) N(x, t)
$$

où $N(x, t)$ désigne le nombre de porteurs créés par unité de volume et $\left(\frac{1}{3} \frac{\mathrm{d} E_{\mathrm{G}}}{\mathrm{d} P}\right)$ une constante qui rend compte de la déformation de la bande interdite du matériau.

Soit la contrainte :

$$
\sigma=\rho v^{2} e_{\mathrm{M}}-E_{\mathrm{el}}\left(\frac{1}{3} \frac{\mathrm{d} E_{\mathrm{G}}}{\mathrm{d} P}\right) N(x, t)
$$

En portant cette équation dans celle du mouvement (7) et en raisonnant d'une manière analogue au cas du couplage thermique, on obtient :

$$
v^{2} \frac{\partial^{2} u(x, t)}{\partial x^{2}}+\omega^{2} u(x, t)=\frac{E_{\mathrm{el}}}{\rho}\left(\frac{1}{3} \frac{\mathrm{d} E_{\mathrm{G}}}{\mathrm{d} P}\right) \frac{\partial N(x, t)}{\partial x}
$$

et pour le déplacement sur la face arrière de l'échantillon en $x=d_{0}$.

$$
u\left(d_{0}\right)_{\mathrm{E}}=\frac{A \lambda_{\mathrm{E}^{2}}}{d_{0}} \quad \text { où } \quad \mid \begin{aligned}
& A=-\beta \frac{\lambda_{\mathrm{E}}}{v^{2}} \\
& \beta=-\frac{E_{\mathrm{e}}}{\rho}\left(\frac{1}{3} \frac{\mathrm{d} E_{\mathrm{G}}}{\mathrm{d} P}\right) \frac{P_{0}}{E_{0} D_{\mathrm{E}}}
\end{aligned}
$$

soit :

$$
\left|u\left(d_{0}\right)\right|_{\mathrm{E}}=\frac{\beta}{v^{2} d_{0}}\left|\sqrt{\frac{D_{\mathrm{E}} \tau}{1+i \omega \tau}}\right|^{3}
$$

Avec les expressions de $\left|u\left(d_{0}\right)\right|_{\mathrm{E}}$ et $\left|u\left(d_{0}\right)\right|_{\mathrm{T}}$, nous allons pouvoir étudier et simuler l'amplitude du déplacement résultant sur la face arrière de l'objet. 


\section{Influence des principaux paramètres sur l'amplitude du signal acoustique.}

Dans les équations (16) et (20) apparaissent plusieurs paramètres comme par exemple la durée de vie $\tau$ des porteurs minoritaires ou les vitesses de diffusion thermique $\left(D_{\mathrm{T}}\right)$ ou électronique $\left(D_{\mathrm{E}}\right)$. A chaque type d'échantillon vont correspondre des valeurs différentes de ces paramètres.

Les courbes des figures 3 et 4 décrivent les variations des quantités $\left|u\left(d_{0}\right)\right|_{\mathrm{T}}$ (Eq. (16)) et $\left|u\left(d_{0}\right)\right|_{\mathrm{E}}$ (Eq. (20)), c'est-à-dire l'amplitude du signal acoustique dû au phénomène thermique ou électronique, en fonction de la fréquence de modulation $f$ du faisceau incident.

L'analyse rapide de ces différents graphes amène plusieurs commentaires.

Tout d'abord, la gamme de fréquence de modulation faisceau utilisée a une importance capitale sur l'origine du phénomène physique dominant la génération d'ultrasons. Ceci est clairement mis en évidence figure 3 où pour $f<100 \mathrm{~Hz}$ (Fig. 3a) l'origine thermique du phénomène est prépondérante alors que lorsque $f$ augmente et devient supérieure à $100 \mathrm{~Hz}$ (Fig. 3b), ce phénomène s'atténue au profit de l'origine électronique de génération d'ultrasons. De plus, l'influence de la durée de vie $\tau$ des porteurs minoritaires est importante. Nous voyons par exemple en comparant les graphes $3 d$ ) et $4 d$ ) que pour une même fréquence de modulation, le phénomène physique dominant est différent.

La figure 5 montre les variations de $\left|u\left(d_{0}\right)\right|_{\mathrm{T}}$ et $\left|u\left(d_{0}\right)\right|_{\mathrm{E}}$ pour différentes valeurs de la durée de vie $\tau$ des porteurs minoritaires pour trois fréquences de modulation faisceau. Cette figure confirme les remarques précédentes et montre l'influence de $\tau$ sur l'amplitude des ultrasons.

Pour chaque graphe de figures 3 et 4 , nous avons déterminé la dépendance en fréquence $f^{\alpha}$ du signal acoustique pour les différents phénomènes à l'origine de la création d'ultrasons. Les tableaux suivants résument les résultats obtenus :

Tableau I. - Dépendance en fréquence $\left|u\left(d_{0}\right)\right|=\mathrm{Cte} \cdot f^{\alpha}$ du signal acoustique pour les différents phénomènes à l'origine de la génération d'ultrasons ( $\tau=10 \mathrm{~ns}$ ).

$\left[\left|u\left(d_{0}\right)\right|=\right.$ Cte $\cdot f^{\alpha}$ Frequency dependence of the acoustic signal for different phenomena of ultrasound generation $(\tau=10 \mathrm{~ns})$.]

\begin{tabular}{|c|c|c|c|c|c|}
\cline { 2 - 6 } \multicolumn{1}{c|}{} & $1-100 \mathrm{~Hz}$ & $100-1 \mathrm{kHz}$ & $1 \mathrm{k}-10 \mathrm{kHz}$ & $10 \mathrm{k}-100 \mathrm{kHz}$ & $100 \mathrm{k}-1 \mathrm{MHz}$ \\
\hline $\begin{array}{c}\text { Phénomène } \\
\text { électronique }\end{array}$ & ind $^{\mathrm{t}}$ & ind $^{\mathrm{t}}$ & ind $^{\mathrm{t}}$ & ind $^{\mathrm{t}}$ & ind $^{\mathrm{t}}$ \\
\hline $\begin{array}{c}\text { Phénomène } \\
\text { thermique }\end{array}$ & $f^{-3 / 2}$ & $f^{-3 / 2}$ & $\approx f^{-3 / 2}$ & $f^{-1}$ & $f^{-1}$ \\
\hline $\begin{array}{c}\text { Signal } \\
\text { total }\end{array}$ & $f^{-3 / 2}$ & $f^{-3 / 2}$ & $\approx f^{-3 / 2}$ & $f^{-1}$ & $f^{-1}$ \\
\hline $\begin{array}{c}\text { Phénomène } \\
\text { dominant }\end{array}$ & \multicolumn{3}{|c|}{ Thermique «pur» } & Thermique « électronique » \\
\hline
\end{tabular}


Tableau II. - Dépendance en fréquence $\left|u\left(d_{0}\right)\right|=$ Cte . $f^{\alpha}$ du signal acoustique pour les différents phénomènes à l'origine de la génération d'ultrasons ( $\tau=1 \mathrm{~ms}$ ).

$\left[\left|u\left(d_{0}\right)\right|=\right.$ Cte $\cdot f^{\alpha}$ Frequency dependence of the acoustic signal for different phenomena of ultrasound generation $(\tau=1 \mathrm{~ms})$.]

\begin{tabular}{|c|c|c|c|c|c|}
\cline { 2 - 6 } \multicolumn{1}{c|}{} & $1-100 \mathrm{~Hz}$ & $100-1 \mathrm{kHz}$ & $1 \mathrm{k}-10 \mathrm{kHz}$ & $10 \mathrm{k}-100 \mathrm{kHz}$ & $100 \mathrm{k}-1 \mathrm{MHz}$ \\
\hline $\begin{array}{c}\text { Phénomène } \\
\text { électronique }\end{array}$ & ind $^{\mathrm{t}}$ & $f^{-3 / 2}$ & $f^{-3 / 2}$ & $f^{-3 / 2}$ & $f^{-3 / 2}$ \\
\hline $\begin{array}{c}\text { Phénomène } \\
\text { thermique }\end{array}$ & $f^{-1}$ & $f^{-5 / 2}$ & $f^{-5 / 2}$ & $f^{-3 / 2}$ & $f^{-3 / 2}$ \\
\hline $\begin{array}{c}\text { Signal } \\
\text { total }\end{array}$ & $f^{-1}$ & $f^{-3 / 2}$ & $f^{-3 / 2}$ & $f^{-3 / 2}$ & $f^{-3 / 2}$ \\
\hline $\begin{array}{c}\text { Phénomène } \\
\text { dominant }\end{array}$ & Thermique & \multicolumn{3}{|c|}{ Electronique «pur » } \\
\hline
\end{tabular}

L'analyse de ces tableaux nous permet de dégager des expressions mathématiques simplifiées du déplacement $u\left(d_{0}\right)$ sur la face arrière de l'échantillon. Ce déplacement détermine directement le niveau de signal utilisable pour la chaîne de mesure.

Les fréquences de modulation faisceau qui nous intéressent dans le cadre de notre étude se situent au-delà de $10 \mathrm{kHz}$. Dans le cas contraire, le pouvoir de résolution de la méthode est trop faible $[3,4]$.

Pour de grandes valeurs de $\tau(\sim 1 \mathrm{~ms}),(20)$ devient :

$$
\left|u\left(d_{0}\right)\right|_{\mathrm{E}}=\frac{E_{\mathrm{el}}}{\rho v^{2} d_{0}}\left(\frac{1}{3} \frac{\mathrm{d} E_{\mathrm{G}}}{\mathrm{d} P}\right) \frac{P_{0}}{E_{0} D_{\mathrm{E}}}\left|\frac{D_{\mathrm{E}} \tau}{1+i \omega \tau}\right|^{3 / 2}=\frac{E_{\mathrm{el}}}{\rho v^{2} d_{0}}\left(\frac{1}{3} \frac{\mathrm{d} E_{\mathrm{G}}}{\mathrm{d} P}\right) \frac{P_{0}}{E_{0} D_{\mathrm{E}}}\left|\frac{D_{\mathrm{E}}}{i \omega}\right|^{3 / 2}
$$

soit :

$$
\left.\left|u\left(d_{0}\right)\right|_{\mathrm{E}}=\frac{E_{\mathrm{el}}}{\rho v^{2}}\left(\frac{1}{3} \frac{\mathrm{d} E_{\mathrm{G}}}{\mathrm{d} P}\right) \frac{1}{E_{0}} \cdot \frac{P_{0} \sqrt{D_{\mathrm{E}}}}{d_{0} \omega^{3 / 2}}\right)
$$

Dans ce cas, le phénomène électronique est dominant et le signal total varie en $f^{-3 / 2}$ Pour des valeurs de $\tau$ plus petites $(\sim 10 \mathrm{~ns})$ c'est le phénomène thermique qui domine et plus particulièrement sa composante électronique. L'équation (16) donne :

$$
\begin{aligned}
\left|u\left(d_{0}\right)\right|_{\mathrm{T}} & =\frac{\alpha E_{\mathrm{e} 1}}{\rho v^{2} d_{0}}\left|\frac{-a}{b+i c}\left[\left(\frac{D_{\mathrm{E}} \tau}{1+i \omega \tau}\right)^{3 / 2}-\left(\frac{D_{\mathrm{T}}}{i \omega}\right)^{3 / 2}\right]+h\left(\frac{D_{\mathrm{T}}}{i \omega}\right)^{3 / 2}\right| \\
& =\frac{\alpha E_{\mathrm{e} 1}}{\rho v^{2} d_{0}}\left|\frac{-a}{b+i c}\left[\left(D_{\mathrm{E}} \tau\right)^{3 / 2}-\left(\frac{D_{\mathrm{T}}}{i \omega}\right)^{3 / 2}\right]+h\left(\frac{D_{\mathrm{T}}}{i \omega}\right)^{3 / 2}\right| .
\end{aligned}
$$



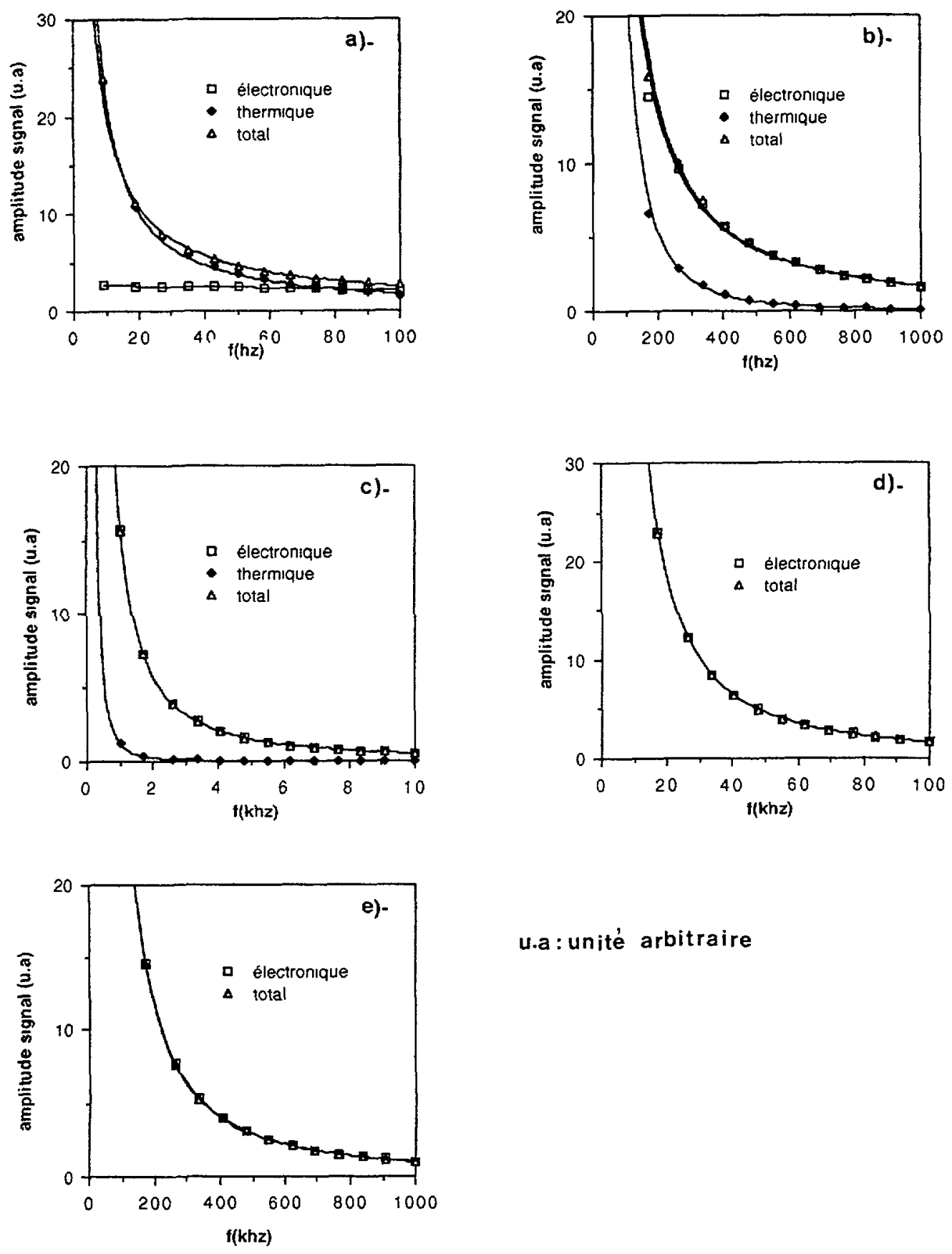

u.a: unité arbitraire

Fig. 3. - Variations de $\left|u\left(d_{0}\right)\right|_{\mathrm{T}},\left|u\left(d_{0}\right)\right|_{\mathrm{E}}$ et $\left|u\left(d_{0}\right)\right|_{\text {Total }}$ en fonction de la fréquence de modulation faisceau $f\left(\tau=1 \mathrm{~ms}, d_{0}=1 \mathrm{~mm}\right.$, échantillon type $\mathrm{P}$ ).

[Variation of $\left|u\left(d_{0}\right)\right|_{\mathrm{T}},\left|u\left(d_{0}\right)\right|_{\mathrm{E}}$ and $\left|u\left(d_{0}\right)\right|_{\text {Total }}$ ws. beam modulation frequency.] 

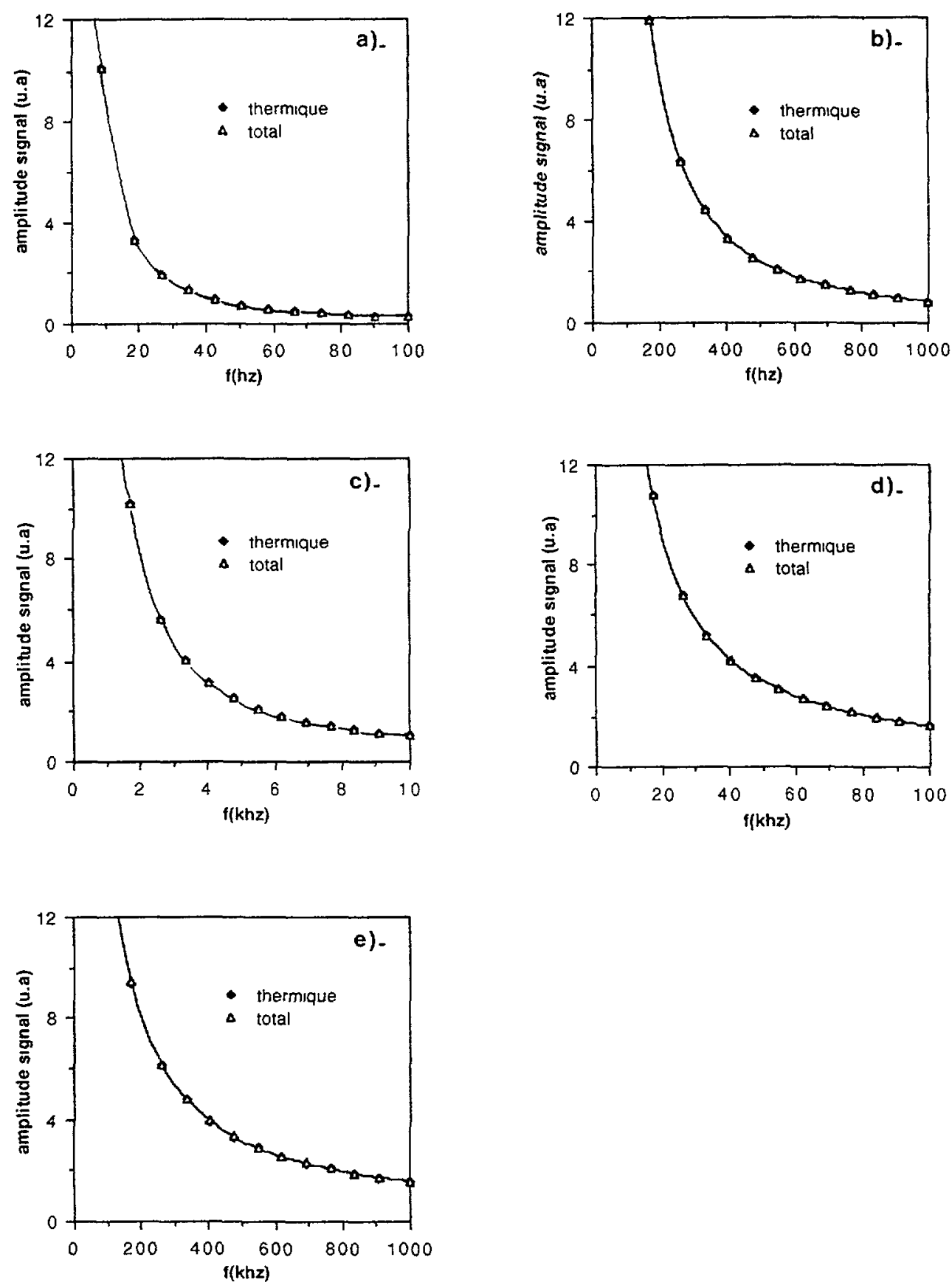

Fig. 4. - Variations de $\left|u\left(d_{0}\right)\right|_{\mathrm{T}},\left|u\left(d_{0}\right)\right|_{\mathrm{E}}$ et $\left|u\left(d_{0}\right)\right|_{\text {Total }}$ en fonction de la fréquence de modulation faisceau $f\left(\tau=10 \mathrm{~ns}, d_{0}=1 \mathrm{~mm}\right.$, échantillon type $\left.\mathrm{P}\right)$.

[Variation of $\left|u\left(d_{0}\right)\right|_{\mathrm{T}},\left|u\left(d_{0}\right)\right|_{\mathrm{E}}$ and $\left|u\left(d_{0}\right)\right|_{\text {Total }}$ vs. beam modulation frequency.] 

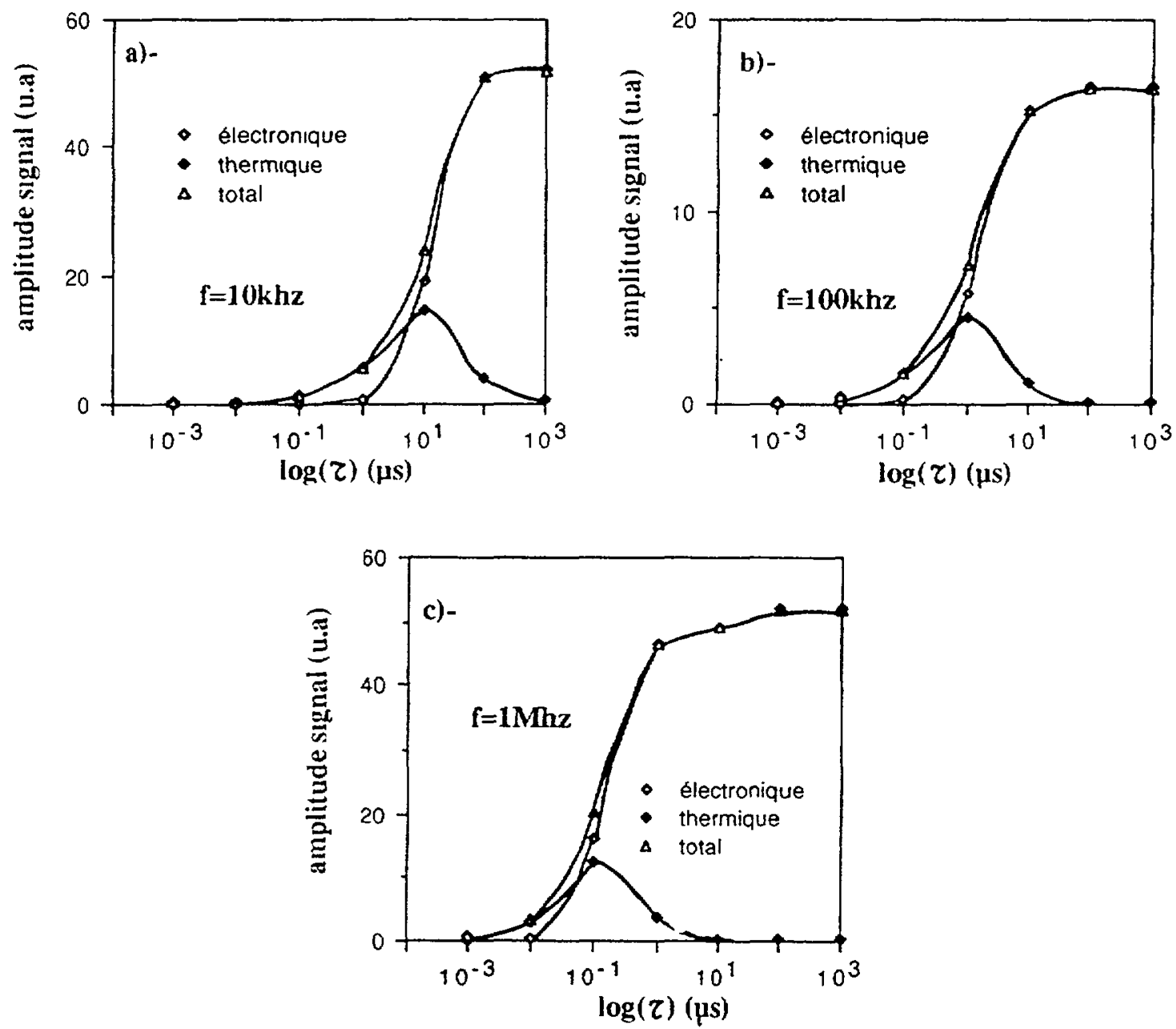

Fig. 5. - Variations de $\left|u\left(d_{0}\right)\right|_{\mathrm{T}},\left|u\left(d_{0}\right)\right|_{\mathrm{E}}$ et $\left|u\left(d_{0}\right)\right|_{\text {Total }}$ en fonction de $\tau$ à $f=10 \mathrm{kHz}$, $f=100 \mathrm{kHz}$ et $f=1 \mathrm{MHz}$.

[Variation of $\left|u\left(d_{0}\right)\right|_{\mathrm{T}},\left|u\left(d_{0}\right)\right|_{\mathrm{E}}$ and $\left|u\left(d_{0}\right)\right|_{\text {Total }} v s . \tau$ at $f=10 \mathrm{kHz}, f=100 \mathrm{kHz}$ and $f=1 \mathrm{MHz}$.]

Une évaluation numérique de ces différents termes amène une nouvelle forme de $\left|u\left(d_{0}\right)\right|_{\mathrm{T}}$.

$$
\left|u\left(d_{0}\right)\right|_{\mathrm{T}}=\frac{\alpha E_{\mathrm{el}}}{\rho v^{2} d_{0}}\left|\frac{-a}{b+i c}\left(D_{\mathrm{E}} \tau\right)^{3 / 2}\right|=\frac{\alpha E_{\mathrm{el}}}{\rho v^{2} d_{0}}\left|\frac{P_{0} E_{\mathrm{G}}\left(D_{\mathrm{E}} \tau\right)^{3 / 2}}{E_{0} D_{\mathrm{E}} \tau K i \omega\left(-1 / D_{\mathrm{T}}\right)}\right|
$$

soit :

$$
\left|u\left(d_{0}\right)\right|_{\mathrm{T}}=\frac{\alpha E_{\mathrm{el}}}{\rho^{2} v^{2} c}\left(\frac{P_{0} E_{\mathrm{G}}}{E_{0}}\right) \frac{\sqrt{D_{\mathrm{E}} \tau}}{d_{0} \omega}
$$


Le tableau III permet de conclure simplement pour des fréquences de travail supérieures à $10 \mathrm{kHz}$ :

Tableau III. - Expression de $\left|u\left(d_{0}\right)\right|$ pour des faibles et forts $\tau$.

[Expression of $\left|u\left(d_{0}\right)\right|$ for low and heavy $\tau_{\text {.] }}$

\begin{tabular}{|l|c|}
\cline { 2 - 3 } \multicolumn{1}{c|}{} & $f>10 \mathrm{kHz}$ \\
\hline Fort $\tau(\geqslant 100 \mu \mathrm{s})$ & $\left|u\left(d_{0}\right)\right|=\mathrm{Cte}\left(\frac{P_{0}}{d_{0}}\right) D_{\mathrm{E}}^{1 / 2} \cdot \frac{1}{f^{3 / 2}} \quad$ Cte $=\frac{E_{\mathrm{el}}}{(2 \pi)^{3 / 2} \rho v^{2}}\left(\frac{1}{3} \frac{\mathrm{d} E_{\mathrm{G}}}{\mathrm{d} P}\right) \cdot \frac{1}{E_{0}}$ \\
\hline Faible $\tau(<1 \mu \mathrm{s})$ & $\left|u\left(d_{0}\right)\right|=\operatorname{Cte}\left(\frac{P_{0}}{d_{0}}\right)\left(D_{\mathrm{E}} \tau\right)^{1 / 2} \cdot \frac{1}{f} \quad$ Cte $=\frac{\alpha E_{\mathrm{el}}}{2 \pi \rho^{2} v^{2} c}\left(\frac{E_{\mathrm{G}}}{E_{0}}\right)$ \\
\hline
\end{tabular}

Ces formules sont importantes en pratique : elles montrent d'une part que suivant la nature de l'échantillon (valeur de $\tau$, niveau de dopage), le phénomène physique à l'origine de la création des ultrasons n'est pas le même. Pour des grandes valeurs de $\tau$, c'est le couplage électronique qui domine et le signal acoustique montre une dépendance en $f^{-3 / 2}$. D'autre part, pour des valeurs de $\tau$ beaucoup plus petites, le couplage thermique domine à travers sa composante électronique, ce qui implique un signal variant en $f^{-1}$.

Dans les deux cas, deux résultats supplémentaires sont à retenir : l'amplitude du signal est inversement proportionnelle à l'épaisseur de l'échantillon $d_{0}$ et directement proportionnelle à la densité de puissance absorbée $\boldsymbol{P}_{0}$.

\section{Application à l'imagerie des défauts dans les semiconducteurs.}

5.1 DÉTECTEur PiÉzoÉlectrique. - Après avoir étudié la génération d'ultrasons dans un échantillon semiconducteur, nous nous proposons de mettre en œuvre la détection des ondes acoustiques. On utilise pour cela un transducteur piézoélectrique (PZT) [4].

Un détecteur PZT typique est constitué d'un élément actif piézoélectrique fixé dans un boîtier dont une paroi sert également de face de couplage. Les caractéristiques du capteur (sensibilité, bande passante) sont fonction de la géométrie et de la partie piézoélectrique.

Le signal électrique à la sortie du détecteur est inversement proportionnel à la surface de contact entre l'échantillon et l'élément piézoélectrique. Ce signal est relié au déplacement sur la face arrière de l'échantillon par la relation [4]:

$$
|V|=\frac{e_{3} L}{\varepsilon_{3}^{s} A}\left|u\left(d_{0}\right)\right|
$$

où :

$e_{3} \quad$ constante du tenseur piézoélectrique $e_{\imath j k}$ du transducteur

$\varepsilon_{3} \quad$ constante du tenseur diélectrique $\varepsilon_{i k}^{\mathrm{s}}$ du transducteur

A surface de contact entre le capteur et l'échantillon

$L \quad$ épaisseur du capteur. 


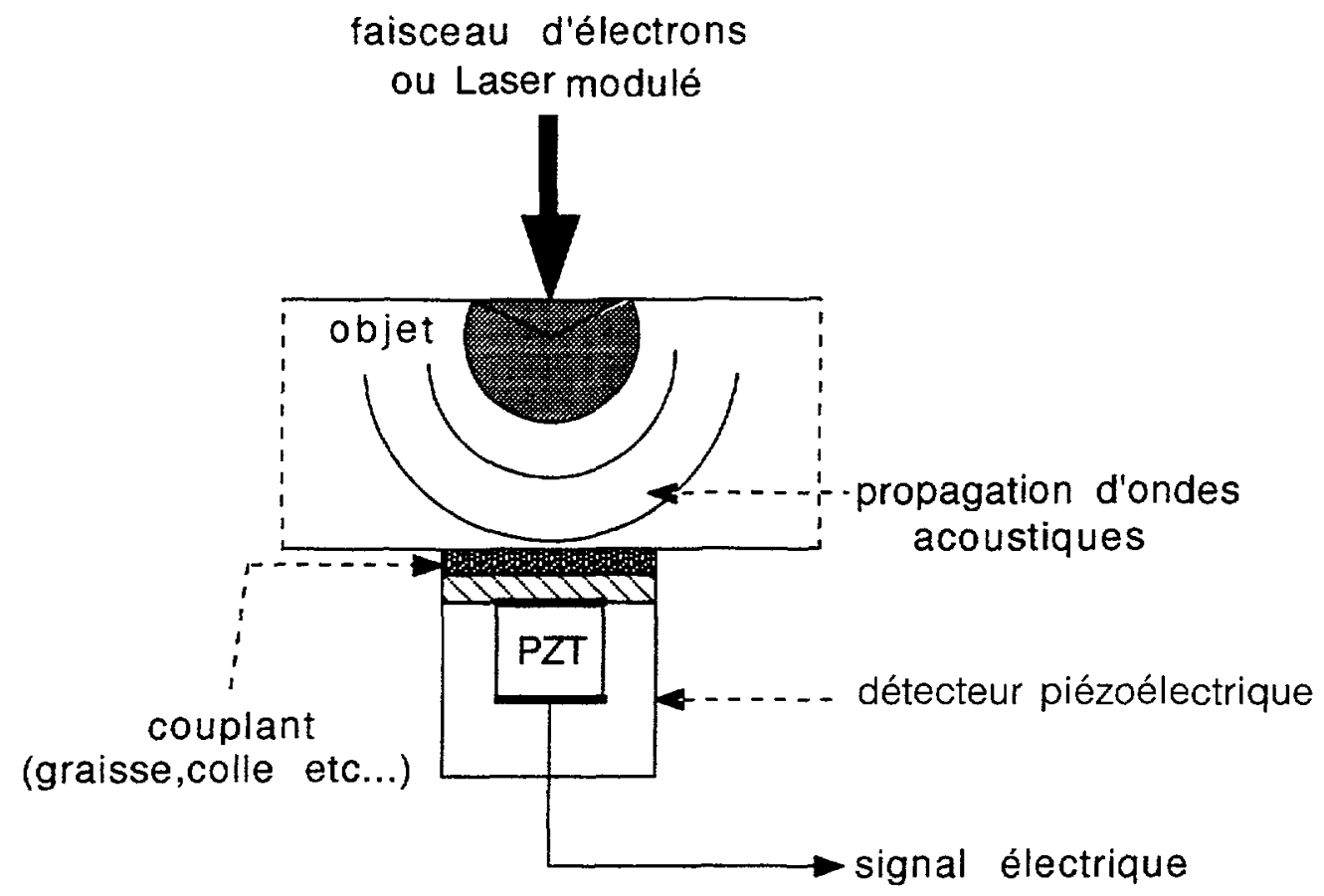

Fig. 6. - Détection des ondes acoustiques à l'aide d'un capteur piézoélectrique.

[Detection of acoustic waves with piezoelectric transducer.]

5.2 Mécanismes de Contraste des images. - Pour réaliser une image de la subsurface d'un échantillon, il faut balayer la surface de l'objet et enregistrer le signal en sortie du capteur point par point. Le contraste observable à l'écran résulte de la variation relative du signal lorsqu'on passe d'un point à un autre.

Les défauts à l'intérieur du volume de génération du signal donnent lieu à des variations de la quantité d'énergie absorbée par le matériau. Ceci peut provoquer une variation du signal enregistré. Mais toute variation locale des propriétés thermiques, électroniques ou élastiques est aussi à l'origine d'un contraste observable.

Pour des matériaux isotropes, les variations éventuelles des constantes élastiques joueront un rôle négligeable dans le mécanisme de contraste [4]. Il faut alors s'intéresser aux variations des propriétés électroniques et thermiques locales à l'intérieur du volume de génération du signal pour pouvoir évaluer ce contraste.

Supposons la présence d'un défaut sous la surface de l'échantillon. Celui-ci permet, par exemple, d'augmenter localement la recombinaison des porteurs.

Si dans la région (1), la durée de vie des porteurs minoritaires est $\tau$, devenant $\tau^{\prime}$ à l'intérieur de la région (2), on peut penser, si $\tau^{\prime} \ll \tau$ (cf. Tab. III) que le signal $V_{1}=V_{1}\left(t_{1}\right)$ n'aura pas la même origine que $V_{2}=V_{2}\left(t_{2}\right)$. Nous obtenons alors:

$$
V_{1} \text {. origine «électronique » }=\operatorname{Cte} \frac{\sqrt{\mathrm{D}_{\mathrm{E}_{1}}}}{\rho_{1}}
$$




\section{Faisceau Laser ou électrons modulé}

\section{Balayage}

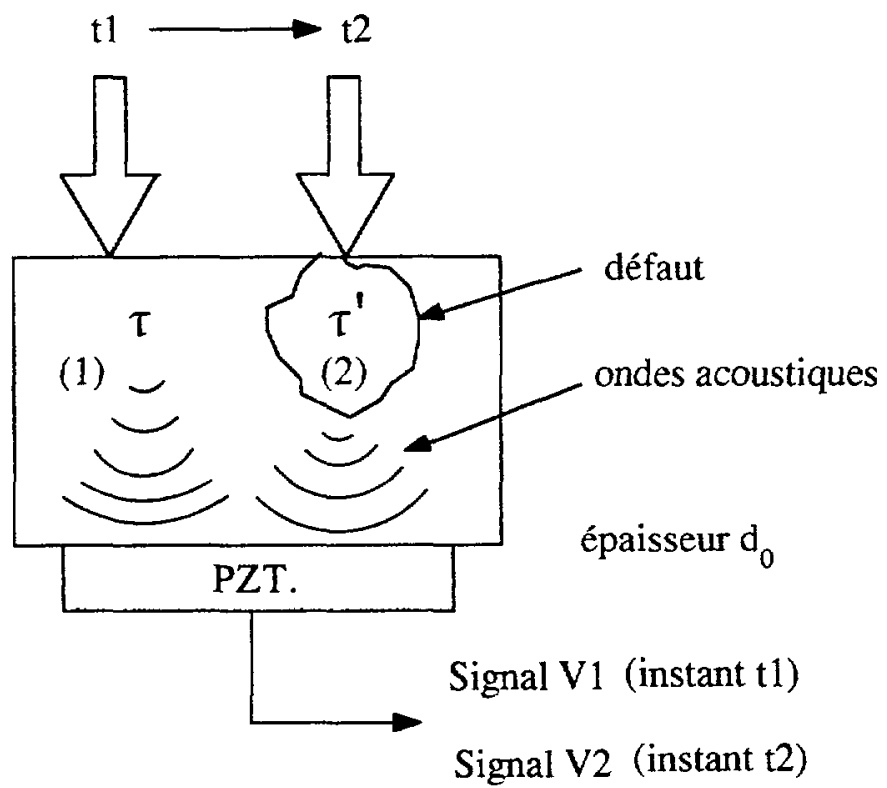

Fig. 7. - Influence d'un défaut en subsurface sur le signal enregistré.

[Influence of a subsurface defect on the recorded signal.]

Le contraste $\Delta V / V$ résulte de la différence des deux niveaux $V_{1}$ et $V_{2}$ et il sera directement lié au phénomène à l'origine de la génération d'ultrasons ainsi qu'aux propriétés électroniques et thermiques du défaut $\left(\alpha_{2}, D_{\mathrm{E}_{2}}\right.$ et $\left.\tau^{\prime}\right)$.

Pour pouvoir observer un constraste dû à la nature de la génération du signal, il sera donc nécessaire que le défaut crée un fort déséquilibre entre $\tau$ et $\tau^{\prime}$.

Dans le cas où $\tau^{\prime} \neq \tau$ mais du même ordre de grandeur, on peut écrire :

$$
\begin{aligned}
& \mid V_{1}=\operatorname{Cte} \sqrt{D_{\mathrm{E}_{1}}} \\
& \mid V_{2}=\operatorname{Cte} \sqrt{D_{\mathrm{E}_{2}}} .
\end{aligned}
$$

Ici, la différence entre $V_{1}$ et $V_{2}$ sera due uniquement à la variation de propriétés électroniques lorsqu'on passe des zones (1) à (2) $\left(D_{\mathrm{E}_{1}} \neq D_{\mathrm{E}_{2}}\right)$. Pour un défaut qui ne crée pas un fort déséquilibre entre $\tau$ et $\tau^{\prime}$, ces variations seront faibles entraînant l'apparition d'un contraste beaucoup moins important que dans le cas précédent.

Dans ce cas, aucun contraste lié à la différence des phénomènes physiques à l'origine de la génération du son ne peut apparaître.

\section{Conclusion.}

Notre étude est proposée dans le cadre de la mise en œuvre d'un dispositif d'imagerie pour la caractérisation des semiconducteurs par faisceau laser. Pour comparer cette méthode avec les résultats déjà obtenus avec le microscope acousto-électronique, nous avons supposé une 
analogie complète faisceau laser-faisceau d'électrons, ce qui se justifie en première approximation car les phénomènes physiques à l'origine de la création d'ondes acoustiques sont les mêmes.

Cette étude nous a permis de dégager des expressions analytiques simples du signal électrique en sortie du capteur piézoélectrique utilisé et de mettre en évidence les paramètres importants qui vont influencer le signal enregistré. Ainsi, suivant la nature de l'échantillon étudié, on pourra connaître les conditions expérimentales optimales à utiliser pour réaliser des images dans de bonnes conditions.

Nous espérons dans un deuxième temps mettre en œuvre une méthode d'analyse temporelle du signal transitoire, ce qui nous permettra d'isoler les signaux correspondant à des niveaux précis et d'obtenir ainsi des «coupes» à différentes profondeurs dans l'échantillon. L'imagerie 3D d'éventuels défauts dans les semiconducteurs sera possible.

\section{Annexe.}

Calcul de $u(x, t)$.

$$
\begin{aligned}
& \sigma(x=0)=0 \Leftrightarrow \rho v^{2} e_{\mathrm{M}}(x=0)=E_{\mathrm{cl}} \alpha T(x=0) \\
&\left.\Leftrightarrow \frac{\partial u(x, t)}{\partial x}\right|_{x=0}=-\beta_{1} \frac{\lambda_{\mathrm{E}}}{v^{2}}-\beta_{2} \frac{\lambda_{\mathrm{T}}}{v^{2}}=A^{\prime}+A^{\prime \prime} \\
& \sigma\left(x=d_{0}\right)=\left.0 \Leftrightarrow \frac{\partial u(x, t)}{\partial x}\right|_{x=d_{0}}=-\left(\beta_{1} \frac{\lambda_{\mathrm{E}}}{v^{2}}\right) \mathrm{e}^{-d_{0} / \lambda_{\mathrm{E}}}-\left(\beta_{2} \frac{\lambda_{\mathrm{T}}}{v^{2}}\right) \mathrm{e}^{-d_{0} \lambda_{\mathrm{T}}} \\
&\left.\Leftrightarrow \frac{\partial u(x, t)}{\partial x}\right|_{x=d_{0}}=A^{\prime} \mathrm{e}^{-d_{0} \lambda_{\mathrm{E}}}+A^{\prime \prime} \mathrm{e}^{-d_{0} / \lambda_{\mathrm{T}}}
\end{aligned}
$$

Les équations (22) et (23) nous donnent le système suivant :

$$
\begin{aligned}
& -i k C_{1}+i k C_{2}-\frac{C_{3}}{\lambda_{\mathrm{E}}}-\frac{C_{4}}{\lambda_{\mathrm{T}}}=A^{\prime}+A^{\prime \prime} \\
& -i k C_{1} \mathrm{e}^{-i k d_{0}}+i k C_{2} \mathrm{e}^{i k d_{0}}-\frac{C_{3}}{\lambda_{\mathrm{E}}} \mathrm{e}^{-d_{0} / \lambda_{\mathrm{E}}}-\frac{C_{4}}{\lambda_{\mathrm{T}}} \mathrm{e}^{-d_{0} / \lambda_{\mathrm{T}}}=A^{\prime} \mathrm{e}^{-d_{0} \lambda_{\mathrm{E}}}+A^{\prime \prime} \mathrm{e}^{-d_{0} \lambda_{\mathrm{T}}}
\end{aligned}
$$

soit :

$$
\begin{array}{r}
-C_{1}+C_{2}=\frac{\left(A^{\prime}+\frac{C_{3}}{\lambda_{\mathrm{E}}}\right)+\left(A^{\prime \prime}+\frac{C_{4}}{\lambda_{\mathrm{T}}}\right)}{i k}-C_{1} \mathrm{e}^{-i k d_{0}}+ \\
+C_{2} \mathrm{e}^{+i k d_{0}}=\frac{\left(A^{\prime}+\frac{C_{3}}{\lambda_{\mathrm{E}}}\right) \mathrm{e}^{-d_{0} \lambda_{\mathrm{E}}}+\left(A^{\prime \prime}+\frac{C_{4}}{\lambda_{\mathrm{T}}}\right) \mathrm{e}^{-d_{0} \lambda_{\mathrm{T}}}}{i k}
\end{array}
$$

d'où :

$$
\begin{aligned}
& C_{1}=\frac{\left(A^{\prime}+\frac{C_{3}}{\lambda_{\mathrm{E}}}\right)\left(\mathrm{e}^{i k d_{0}}-\mathrm{e}^{-d_{0} \lambda_{\mathrm{E}}}\right)+\left(A^{\prime \prime}+\frac{C_{4}}{\lambda_{\mathrm{T}}}\right)\left(\mathrm{e}^{i k d_{0}}-\mathrm{e}^{-d_{d^{\prime}} \lambda_{\mathrm{T}}}\right)}{2 k \sin k d_{0}} \\
& C_{2}=\frac{\left(A^{\prime}+\frac{C_{3}}{\lambda_{\mathrm{E}}}\right)\left(\mathrm{e}^{-\imath k d_{0}}-\mathrm{e}^{-d_{d} \lambda_{\mathrm{E}}}\right)+\left(A^{\prime \prime}+\frac{C_{4}}{\lambda_{\mathrm{T}}}\right)\left(\mathrm{e}^{-i k d_{0}}-\mathrm{e}^{-d_{d^{\prime} \lambda_{\mathrm{T}}}}\right)}{2 k \sin k d_{0}}
\end{aligned}
$$


d'où en reportant dans (12):

$$
\begin{aligned}
& u(x, t)=\left(\frac{\left(A^{\prime}+\frac{C_{3}}{\lambda_{\mathrm{E}}}\right)\left(\mathrm{e}^{i k d_{0}}-\mathrm{e}^{-d_{d} \lambda_{\mathrm{E}}}\right)+\left(A^{\prime \prime}+\frac{C_{4}}{\lambda_{\mathrm{T}}}\right)\left(\mathrm{e}^{i k d_{0}}-\mathrm{e}^{-d_{0} / \lambda_{\mathrm{T}}}\right)}{2 k \sin k d_{0}} \mathrm{e}^{-i k x}+\right. \\
& +\frac{\left(A^{\prime}+\frac{C_{3}}{\lambda_{E}}\right)\left(\mathrm{e}^{-\imath k d_{0}}-\mathrm{e}^{-d_{0} / \lambda_{\mathrm{E}}}\right)+\left(A^{\prime \prime}+\frac{C_{4}}{\lambda_{\mathrm{T}}}\right)\left(\mathrm{e}^{-\imath k d_{0}}-\mathrm{e}^{-d_{0} / \lambda_{\mathrm{T}}}\right)}{2 k \sin k d_{0}} \mathrm{e}^{\imath k x} \\
& \left.+C_{3} \mathrm{e}^{-x / \lambda_{\mathrm{E}}}+C_{4} \mathrm{e}^{-x / \lambda} \mathrm{T}\right) \mathrm{e}^{l \omega t} \\
& =\left(\begin{array}{l}
\frac{\left\{\left(A^{\prime}+\frac{C_{3}}{\lambda_{\mathrm{E}}}\right)+\left(A^{\prime \prime}+\frac{C_{4}}{\lambda_{\mathrm{T}}}\right)\right\} \cos k\left(x-d_{0}\right)-}{-\left\{\left(A^{\prime}+\frac{C_{3}}{\lambda_{\mathrm{E}}}\right) \mathrm{e}^{-d_{0} \lambda_{\mathrm{E}}}+\left(A^{\prime \prime}+\frac{C_{4}}{\lambda_{\mathrm{T}}}\right) \mathrm{e}^{-d_{0} / \lambda_{\mathrm{T}}}\right\} \cos k x}+C_{3} \mathrm{e}^{-x / \lambda_{\mathrm{E}}}+C_{4} \mathrm{e}^{-x / \lambda_{\mathrm{T}}}
\end{array}\right) \mathrm{e}^{i \omega t}
\end{aligned}
$$

\section{Bibliographie}

[1] SILK M. G., «Ultrasonic transducers for non destructive testing», Adam Hilger Ltd. Bristol (1984).

[2] Aussel J. D., Le Brun A., Baboux J. C., «Generating acoustic waves by laser. Theorical and experimental study of the emission source ", Ultrasonics 26 (1988) 245-255.

[3] Franceschi J. L., Murillo R., Bastie A., Ez ZejJari M., «Imagerie acousto-électronique » $J$. Phys. Colloq. France 51 (1990) C2.

[4] Ez ZeJJARI M., Thèse de Doctorat Toulouse (1991).

[5] Opsal J., Rosencwaig A., «Thermal waves depth profiling: theory » J. Appl. Phys. 53 (1982) 4240.

[6] RosenCWAig A., « Thermal waves imaging in scanning electron microscopy ». Scanning Electron Microsc. II (1984) 1611-1628.

[7] BALK L. J., « Scanning electron acoustic microscopy», Ad. Electron. Electron Phys. 71 (Acad. Press, 1988).

[8] Kultscher N., BALK L. J., « Signal generation and contrast mechanisms in Scanning Electron Acoustic Microscopy ", Scanning Electron Microsc. I (1986) 33-43.

[9] Sablikhov V. A., Sandomirski V. B., « The photoacoustic effect in Semiconductors », Phys. Stat. Solidi B 120 (1983) 471.

[10] Stearns R. G., Kino G. S., « Effect of electronic strain on photoacoustic generation in silicon », Appl. Phys. Lett. 47 (1985).

[11] Fournier D., Boccara C., Skumanich A., AMer N. M., «Photothermal investigation of transport in semiconductors : theory and experiment ». J. Appl. Phys. 59 (1986).

[12] BOucher J., Thèse doctorat d'état Toulouse (1974).

[13] AUld B. A., « Acoustic fields and waves in solids». Scanning Electron Microsc. I (1973). 